found in partial or complete androgen insensitivity syndrome. The aim of this study was to determine whether even the most minor forms of isolated hypospadias are associated with AR mutations and thus whether all types of hypospadias warrant molecular analysis of the AR.

Methods 292 Caucasian children presenting with isolated hypospadias (no micropenis, no cryptorchidism) and 345 controls were included prospectively. Mutational analysis of the AR through direct sequencing (exons 1-8) was performed.

Results Five missense mutations of the AR were identified in 9 patients with glandular or penile anterior $(n=5)$, penile midshaft $(n=2)$ and penile posterior $(n=2)$ hypospadias, i.e., 3\%: p.Q58L (c.1288 A>T), 4 cases of p.P392S (c.2289 C>T), 2 cases of p.A475V (c.2539 C>T), p.D551H (c.1651 G>C) and p.Q799E (c.3510 C>G). None of these mutations was present in the control group. Four mutations are novel findings since 1 has never been reported to date (p.D551H) and 3 have never been reported in patients with genital malformation but only in isolated infertility: p.Q58L, p.P392S, and p.A475V. It is notable that micropenis, a cardinal sign of AIS, was not present in any patients.

Conclusion AR mutations may play a role in the cause of isolated hypospadias, even in the most minor forms. Identification of this underlying genetic alteration is important for proper diagnosis and may significantly improve the follow-up of these patients during puberty, especially regarding future fertility.

\section{THE FEASIBILITY OF TISSUE EXPANDERS IN RECONSTRUCTION OF GIANT CONGENITAL MELANOCYTIC NEVI IN CHILDREN}

doi:10.1136/archdischild-2012-302724.1587

'M Fahmy, ${ }^{2}$ AA Mazy. 'Pediatric Surgery, Al Azher University, Cairo; ${ }^{2}$ Anesthesia, Al Masuera University, Masuera, Egypt

Background Children with Giant Congenital Melanocytic Nevi (GCMN) carry a great challenge to the pediatric and reconstructive surgeons to cover the widely exposed area after its excision. A variety of treatment options exists for the management of such cases. In this retrospective review of selected group of children had a GCMN of their abdominal walls were managed with implantation of tissue expanders (TE) for staged reconstruction, patients evaluated with respect to complications, general and esthetic patient and parents satisfaction.

Objective Our purpose was to study the feasibility of use tissue expanders in the management of children who had GCMN with special emphasis to the complications and children and their parents satisfaction.

Material and Methods Retrospective data collection of the 12 patient's charts, operative data of 86 surgical procedures and follow up visits were calculated and analyzed, with. Statistical analysis done using the Student $t$ test, and $\mathrm{P}<0.05$ was considered statistically significant.

Results In a period of 4 years, from 2004 to 2008, the results of using 37 different sizes tissue expanders and 86 operative procedures in a 12 child, 9 boys and 3girls aged from 2 to 12 years with different types of abdominal wall GCMN will be discussed.

Conclusion Tissue expander is a useful and feasible tool for reconstruction of the abdominal wall in cases of GCMN in children. Parents and children satisfaction and body image are generally acceptable.

\section{COMPARISON OF CIRCUMCISIONS PERFORMED WITH PLASTIC CLAMP AND CONVENTIONAL DISSECTION TECHNIQUE}

doi:10.1136/archdischild-2012-302724.1588
FM Senel, F Misirlioglu. Dr. Sami Ulus Hospital, Ankara, Turkey

Background and Aims In the current study we serched the outcome of circumcisions performed with a new plastic clamp. The results were compared to those who underwent conventional circumcision.

Methods We analyzed the short-term complications of the procedure among the 3650 children who were consecutively circumcised by plastic Alis klamp technique. The results were compared to those of 1275 children who were circumcised by conventional technique. Results The rate of infection $(0.4 \%)$ and bleeding $(0.2 \%)$ after plastic clamp were significantly lower compared to those children circumcised by conventional technique (3.2\% and $4.1 \%$; $<0.0001$ ). Buried penis occured in similar rates among both groups. We did not encounter any penile injury in either groups. The total complication rates of plastic clamp and conventional techniques were found as $1.8 \%$ and $8.7 \%$, respectively $(p<0.0001)$. The mean duration of circumcision time with plastic clamp and conventional technique was $4.1 \pm 1.2$ min and $24 \pm 3.6$ min, respectively ( $<<0.0001$ ).

Conclusion Plastic Alis klamp technique is suggested as the circumcision procedure of choice due to reduced complications, as well as short duration and ease of application.

Abstract 1588 Table 1 Comparison of complications

\begin{tabular}{lllllc}
\hline & Plastic clamp (n 3650) & \% & Conventional (n 1275) & $\%$ & p \\
\hline Age (years) & $6.2 \pm 1.5$ & & $6.9 \pm 1.8$ & & \\
Infection & 14 & 0.4 & 41 & $3.2<0.0001$ \\
Bleeding & 7 & 0.2 & 52 & $4.1<0.0001$ \\
Buried penis & 44 & 1.2 & 18 & 1.4 & NS \\
Duration (min) & $4.1 \pm 1.2$ & & $24 \pm 3.6$ & & $<0.0001$ \\
Total complications & 65 & 1.8 & 111 & $8.7<0.0001$ \\
\hline
\end{tabular}

\section{ROLE OF THORACOSCOPY IN THE TREATMENT OF LOCULATED PLEURAL EMPYEMA IN PEDIATRIC PATIENTS: ABOUT 73 CASES}

doi:10.1136/archdischild-2012-302724.1589

${ }^{1} \mathrm{~J}$ Chahed, ${ }^{2} \mathrm{~K}$ Maazoun, ${ }^{2} \mathrm{~S}$ Hidouri, ${ }^{2} \mathrm{~A}$ Ksia, ${ }^{2} \mathrm{~K}$ Krichene, ${ }^{2} \mathrm{~L}$ Sahnoun, ${ }^{2} \mathrm{M}$ Mekki, ${ }^{2}$ A Nouri. ${ }^{1}$ Pediatric Surgery; ${ }^{2}$ EPS Fattouma Bourguiba, Monastir, Tunisia

Objective Evaluate the results of thoracoscopy for the treatment of located pleural empyema.

Methods Retrospective study of 73 patients with loculated pleural empyema in pediatric surgery department of Monastir during the period between 1997-2010. Located empyema was confirmed in all patients through imaging (ultrasonography or tomography of chest).

Results : The age of patients ranged between 2 months- 16 years (mean age: 41.5 months) with a sex ration of 1.28. Fever was present in $98 \%$ of the cases. 25 patients had severe respiratory distress and mechanical ventilation was necessary in 4 cases. Bacteriology of the pleural liquid was positive in 12 cases. All patients had intravenous antibiotherapy during a mean period of 20 days. Sixteen patients $(22 \%)$ were treated by thoracic drainage. Intrapleural fibrolysis was indicated in 5 cases (7\%). One patient had an open thoracotomy for lung abscess with a loculated pleural empyema. Thoracoscopy was performed in 51 cases (70\%). It was indicated after thoracic drainage failure in 14 cases. It was indicated for firstline before any prior thoracic drainage in 37 cases because of a multiloculated aspect at ultrasonography.

Conclusion Management of pleural empyema is still controversal in children and surgical indication is often delayed. Early first-line thoracoscoy yields a better clinical outcome for pediaric patients with pleural loculated empyema, with apparent decreased morbidity, earlier 
apyrexia, earlier chest tube removal, earlier hospital discharge and better response to antibiotherapy. Thoracoscopy prior to thoracic drainage can be indicated as first line treatment of loculated empyema for children.

\section{INGUINAL HERNIA IN “GIRLS” RARELY REVEALS COMPLETE ANDROGEN INSENSITIVITY SYNDROME}

doi:10.1136/archdischild-2012-302724.1590

${ }^{1} \mathrm{~N}$ Kalfa, ${ }^{2} \mathrm{~F}$ Paris, ${ }^{1} \mathrm{~A}$ Andreica, ${ }^{2} \mathrm{~F}$ Audran, ${ }^{1} \mathrm{O}$ Maillet, ${ }^{2 \mathrm{P}}$ Philibert, ${ }^{3} \mathrm{RB}$ Galifer, ${ }^{4} \mathrm{C}$ Sultan. ${ }^{1}$ Pediatric Surgery; ${ }^{2}$ Pediatric Endocrinology; ${ }^{3} \mathrm{CHU}$ Montpellier, Montpellier; ${ }^{4} \mathrm{CHU}$ Montpellier, Paris, France

Background Aim According to literature, the incidence of complete androgen insensitivity syndrome (CAIS) revealed by inguinal hernia in "girls" is variable due to the clinical heterogeneity of the series. The aim of this study is to estimate the percentage of CAIS in children with female phenotype who presented with various forms of hernias.

Material and Methods This is a retrospective study based on a population of 129 "girls" treated for bilateral hernia repair. The gonads were assessed either by preoperative US or by intra operative direct examination. In case of CAIS suspicion, gonadic tissue was sampled, karyotyping and hormonal analysis were performed. Diagnosis of CAIS was confirmed by direct AR gene sequencing (exons 1-8).

Results We identified 2 cases of CAIS (mutations pS204N+delR615 and del F584). The percentage of CAIS depends on the population involved. On the entire series (including simple permeability of the peritoneo-vaginal channel, $n=129$ ), the rate of CAIS is low, $1.6 \%$. In case of clinical bilateral hernia whatever the content, digestive or gonadal, the rate of CAIS climbs at $6.9 \%$. For the bilateral gonadic hernias $(n=7)$, the rate of CAIS is $28.6 \%$.

Conclusions The incidence of CAIS among "girls" undergoing bilateral hernia repair is low and varies according to the involved population. The simple permeability of the contralateral channel is not a significant risk factor for CAIS. Systematic research of CAIS may be justified in a small number of patients, especially those with bilateral gonadal content. Visualization of the gonads remains mandatory in these particular patients.

\section{DETECTION OF HERPES VIRUSES IN CHILDREN WITH ACUTE APPENDICITIS}

doi:10.1136/archdischild-2012-302724.1591

'E Blevrakis, 'C Seremeti, ${ }^{2 P}$ Katzoli, ${ }^{2 M}$ Ergazaki, ${ }^{2} \mathrm{DA}$ Spandidos, ${ }^{2} \mathrm{G}$ Sourvinos, ${ }^{1} \mathrm{G}$ Sakellaris. 'Department of Paediatric Surgery, University Hospital of Heraklion, Crete; ¿Laboratory of Virology, Faculty of Medicine, University of Crete, Heraklion, Greece

Objective This study aimed to investigate the incidence of HSV types-1 and -2, VZV, CMV, EBV, HHV-6 and HHV-7 in childhood acute appendicitis.

Study Design Polymerase chain reaction (PCR) assays were applied to detect herpes virus DNA in 38 children [11 girls and 27 boys, mean age 9 years (STD \pm 2.59 ), range 6-14 years], who underwent an appendectomy within a 2.5 -year period. Appendix, omentum and peripheral blood mononuclear cells (PBMCs) were available from each case. Of the 38 children with acute appendicitis, $20(52.6 \%)$ had advanced (phlegmonous) acute appendicitis and $18(47.4 \%)$ had perforated appendicitis and local peritonitis. Fortyone blood specimens from age-matched healthy children (25 female and 16 male), with clinical manifestations unrelated to viral infections served as negative controls.

Results CMV was the most frequently detected virus (8/38.21\%), followed by HHV-6 (3/38.7.9\%). EBV and HSV-1 were detected, though not in all three different types of tissue specimens tested. None of the samples examined were HSV-2, VZV or HHV-7 positive.
Of all the specimens, the omentum was the most commonly infected tissue $(63.0 \%)$ while the appendix and peripheral blood specimens were found to be positive for viral infection in $60.5 \%$ and $50 \%$ of cases, respectively. The CMV IgG+ antibodies were positive in $54 \%$ of the control cases while $86 \%$ of the same group presented HHV-6 IgG+ antibodies.

Conclusion To the best of our knowledge, this is the first study documenting the presence of herpes virus DNA in children with acute appendicitis, suggesting that possible viral infection or reactivation is associated with childhood appendicitis.

\section{SACROCOCCYGEAL TERATOMA: 10 YEARS EXPERIENCE IN UPPER EGYPT}

doi:10.1136/archdischild-2012-302724.1592

'MA Osman, ${ }^{2} \mathrm{IA}$ Ibrahim. 'Pediatric Surgical Unit, Surgery Department; ${ }^{2}$ Pediatric Surgery Unit, Faculty of Medicine, Assiut University, Assiut, Egypt

Purpose To evaluate our experience of 45 patients with SCT (in Upper Egypt) over a period of 10 years (2001-2011), and to determine of the outcome of the management and recommendations for treatment's strategies.

Patients and Methods The records were reviewed for age at presentation, clinical manifestations and investigations, time of surgical approach, histopathology, recurrences, bladder and anorectal function, and cosmetic outcome.

Results The time of referral was immediately after birth in 5 patients, at the $1^{\text {st }}$ week in 17 , later in infancy in 22 , and at age of 1.5 years in one child. Excision of the lesion was done for 41 patients. Teratomas were type I $(n=9)$, type II $(n=20)$, type III $(n=11)$, type IV $(n=1)$ (Altman's classification). The age of operation ranged from 2 days to 1.5 years. Histology of results were: mature teratoma $(n=27)$, immature teratoma $(n=9)$, malignant teratoma $(n=5)$. Coccyx remained in two cases of early period of study.

FU ranged from 3 months to 10 years. Recurrence rate in 13 $(31.7 \%)$, wound infection occurred in $4(9.7 \%)$, diarrhea occurred in 2 (4.8\%). AFP was high in 35 and normal in two patients, it decreased after excision. Fetal diagnosis was made in 5 cases by prenatal sonography.

Conclusion Prenatal diagnosis of SCT is important and recommended to save the baby from obstructed labor. Early diagnosis allows early surgical intervention avoids malignant transformation. The coccyx should be excised to decrease the risk of recurrence. Skin flap modification is feasible for large teratomas with healthy skin.

\section{ANTENATALLY DIAGNOSED OVARIAN CYSTS: MANAGEMENT AND FOLLOW-UP PROTOCOL}

doi:10.1136/archdischild-2012-302724.1593

'F Laconi, 'V Guerriero, ${ }^{2} \mathrm{R}$ Raffaelli, ${ }^{3} \mathrm{M}$ Carli, ${ }^{4} \mathrm{FS}$ Camoglio. 'Pediatric Surgery; ${ }^{2}$ Ultrasound and Prenatal Diagnosis Service UO Obstetrics Gynecology OP, University of Verona, Policlinico G.B. Rossi; ${ }^{3}$ Ultrasound and Prenatal Diagnosis Service UO Obstetrics Gynecology OP, ${ }^{4}$ Pediatric Surgery, University of Verona, Policlincio G.B. Rossi, Verona, Italy

Objectives Abdominal cysts are frequently detected in fetuses through routine prenatal ultrasound.

In female fetuses such cysts are most often originated from ovaries under the stimulus of maternal hormones, and can grow to a rather large size

We present our survey of prenatally detected ovarian cysts (OC) and we propose a management and follow-up protocol.

Methods A retrospective review of all cases of abdominal cysts in female fetuses detected in our institution from jan. 2007 to jan. 2012 was conducted.

Among all cases of abdominal cystic formations, 28 were originated from the ovary, and resulted at antenatal US scan ranging in 